\title{
Low Temperature and Radiation Stability of Flexible IGZO TFTs and their Suitability for Space Applications
}

\author{
Júlio C. Costa ${ }^{1}$, Arash Pouryazdan ${ }^{1}$, Julianna Panidi ${ }^{2}$, Thomas Anthopoulos ${ }^{2,3}$, Maciej O. Liedke ${ }^{4}$, Christof \\ Schneider $^{4}$, Andreas Wagner ${ }^{4}$, Niko Münzenrieder ${ }^{1}$
}

${ }^{1}$ University of Sussex, Brighton, United Kingdom, ${ }^{2}$ Imperial College, London, United Kingdom, ${ }^{3}$ King Abdullah University of Science and Technology, Thuwal, Saudi Arabia, ${ }^{4}$ Helmholtz-Zentrum Dresden-Rossendorf, Dresden, Germany

\begin{abstract}
In this paper, Low Earth Orbit radiation and temperature conditions are mimicked to investigate the suitability of flexible Indium-Gallium-Zinc-Oxide transistors for lightweight space-wearables. Such wearable devices could be incorporated into spacesuits as unobtrusive sensors such as radiation detectors or physiological monitors. Due to the harsh environment to which these space-wearables would be exposed, they have to be able to withstand high radiation doses and low temperatures. For this reason, the impacts of high energetic electron irradiation with fluences up to $10^{12} \mathrm{e}^{-/ \mathrm{cm}^{2}}$ and low operating temperatures down to $78 \mathrm{~K}$, are investigated. This simulates $278 \mathrm{~h}$ in a Low Earth Orbit. The threshold voltage and mobility of transistors that were exposed to $\mathrm{e}^{-}$irradiation are found to shift by $+0.09 \pm 0.05 \mathrm{~V}$ and $-0.6 \pm 0.5 \mathrm{~cm}^{2} \mathrm{~V}^{-1} \mathrm{~s}^{-1}$. Subsequent low temperature exposure resulted in additional shifts of $+0.38 \mathrm{~V}$ and $-5.95 \mathrm{~cm}^{2} \mathrm{~V}^{-1} \mathrm{~s}^{-1}$ for the same parameters. These values are larger than the ones obtained from non-irradiated reference samples. If this is considered during the systems' design, these devices can be used to unobtrusively integrate sensor systems into space-suits.
\end{abstract}

Keywords-flexible electronics; space applications; amorphous oxides; wearables; thin film transistors.

\section{INTRODUCTION}

The development of electronic devices for space applications is a widely researched topic since the beginning of the space age. Due to the extreme conditions present outside the Earth's atmosphere, specialized electronic equipment and shielding are required to make these devices capable of withstanding large temperature variations, as well as constant doses of radiation. As an example, special considerations have to be taken when developing instrumentation for the International Space Station, as it regularly passes through the South Atlantic Anomaly (SAA) [1]. This area is characterized by an increase in radiation and energized charged particles due to the weak local geomagnetic field - in particular, the flux of energized electrons (energies up to $5 \mathrm{MeV}$ ) can reach $10^{6} \mathrm{e}^{-} \mathrm{cm}^{-2} \mathrm{~s}^{-1}$ [2], [3]. The interaction between these electrons and electronic systems causes failures due to ionization effects and atomic displacements in the bulk of semiconductors, which has been prevented by the implementation of bulky shielding structures. As space travels become more common and more sensors are required to ensure the astronauts' safety, the development of

This work was partially supported by EPSRC, GCRF, and NIHR, under the contact number: EP/R013837/1 (SmartSensOtics)

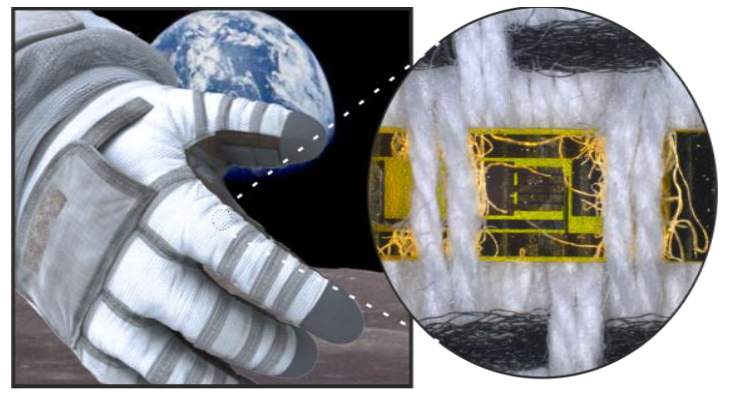

Fig. 1. Concept of an a-IGZO circuit incorporated into a spacesuit. This material could be used for the development of unobtrusive systems that measure e.g. temperature and radiation from the environment or even physiological data from the astronaut.

lightweight and robust electronic devices is required. In this context, flexible electronics are a viable option for the development of future space suits. Fig. 1 shows a vision for spacesuit-integrated textile electronics. Amorphous oxide materials such as Indium-Gallium-Zinc-Oxide (a-IGZO) [4] would be an adequate candidate for the development of conformable and lightweight, yet high performance, physiological sensor systems. [5]. Furthermore, its amorphous phase improves its radiation hardness since there is no crystalline structure to be damaged. To apply this semiconductor on space wearable applications, its suitability and stability must be assessed. While the mechanical stability of a-IGZO has already been extensively studied by demonstrating outstanding bending stability down to $25 \mu \mathrm{m}$ bending radii [6]-[9], low temperature, and electron irradiation stress is equally important for space applications. Previously, it was shown that rigid a-IGZO transistors continue to operate after being exposed to relatively low energetic electron irradiation (0.8 MeV - $\left.10^{14} \mathrm{e}^{-} / \mathrm{cm}^{2}\right)$ [10]. However, the employed bulky and rigid substrates interact with the energized electrons and can shield the semiconductor channel. Simultaneously, the electrons in the SAA are more energetic. Hence, these results cannot be used to predict the response of devices on flexible and thin polymer substrates. Similarly, it is known that a-IGZO can function at low temperatures. However, due to the larger thermal expansion coefficient of deformable polymer foils compared to 


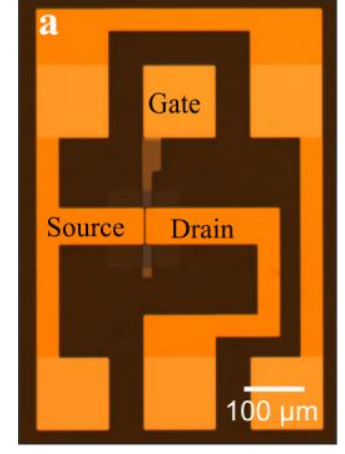

b

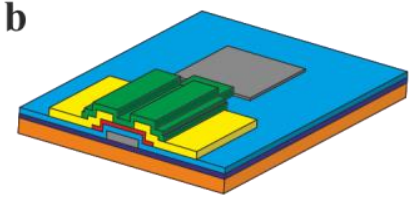

a-IGZO semiconductor $(15 \mathrm{~nm})$

$\square$ Cr gate $(35 \mathrm{~nm})$

$\square \mathrm{Al}_{2} \mathrm{O}_{3}$ dielectric $(25 \mathrm{~nm})$

$\square \mathrm{Ti}(10 \mathrm{~nm}) / \mathrm{Au}(60 \mathrm{~nm})$ Contacts

- $\mathrm{Al}_{2} \mathrm{O}_{3}$ passivation $(25 \mathrm{~nm})$

$\square$ Polyimide substrate $(50 \mu \mathrm{m})$

- Silicon Nitride adhesion layer $(50 \mathrm{~nm})$

Fig. 2. a) Micrograph of the transistors studied in this work. (b) Schematic of the staggered bottom gate transistors.

rigid substrates, it is necessary to characterize the temperature stability of flexible a-IGZO thin film transistors (TFTs) [11].

In this study, flexible a-IGZO transistors are characterized before and after exposure to electrons with an energy of $34.1 \mathrm{MeV}$ and electron fluences spanning 6 orders of magnitude, followed by the characterization of their operation for temperatures down to $78 \mathrm{~K}$, which is below the minimum temperature measured for shaded objects in Low Earth Orbit [12]. It is demonstrated that these flexible devices remained fully operational after being irradiated by $34.1 \mathrm{MeV}$ electrons with an electron density of $10^{12} \mathrm{e} / \mathrm{cm}^{2}$, followed by the exposure to $78 \mathrm{~K}$. These measurements simulate a $278 \mathrm{~h}$ spacewalk above the South Atlantic Anomaly, which is sufficiently longer than typical spacewalks.

\section{DEVICE FABRICATION \& CHARACTERISATION}

Fig. 2a shows a micrograph of a characterised transistor sample. All a-IGZO TFTs were fabricated on a free-standing $50 \mu \mathrm{m}$ thick polyimide foil (Fig. 2b). To improve adhesion, a $50 \mathrm{~nm}$ thick silicon nitride layer was deposited through plasma-enhanced chemical vapor deposition. The gate consists of a $35 \mathrm{~nm}$-thick $\mathrm{Cr}$ layer deposited through e-beam evaporation. The $25 \mathrm{~nm}$ thick $\mathrm{Al}_{2} \mathrm{O}_{3}$ gate insulator layer was deposited through atomic layer deposition (ALD) at a temperature of $150{ }^{\circ} \mathrm{C}$. Afterwards, a $15 \mathrm{~nm}$ IGZO layer was deposited by RF-magnetron sputtering at room temperature. The drain and source contacts were fabricated by depositing $10 \mathrm{~nm}$ of titanium and $60 \mathrm{~nm}$ of gold through e-beam evaporation. A subsequent $25 \mathrm{~nm}$ thick ALD $\mathrm{Al}_{2} \mathrm{O}_{3}$ layer was deposited to passivate the transistors. The devices were measured using a Keysight B1500A parameter analyser. Electron irradiation was performed at a direct-beam end-station at the superconducting electron LINAC ELBE [13] at HZDR. The electron fluence calibration has been performed by measuring the electric current in a Faraday cup and the dose rate in an ionization chamber Roos model 34001 [14]. We assume an error of the fluence measurement of max. $\pm 10 \%$. Electrical characterization of the devices at low temperatures was carried out under vacuum $\left(10^{-5}\right.$ mbar) at temperatures varying from $77 \mathrm{~K}$ to $300 \mathrm{~K}$ using a cryogenic probe station (Janis Research, ST-500) and an Agilent B2902A source measure unit. All measurements were conducted in the dark, on transistors originating from the same substrate. Performance parameters were extracted from the saturation regime using the Shichman-Hodges model [15].
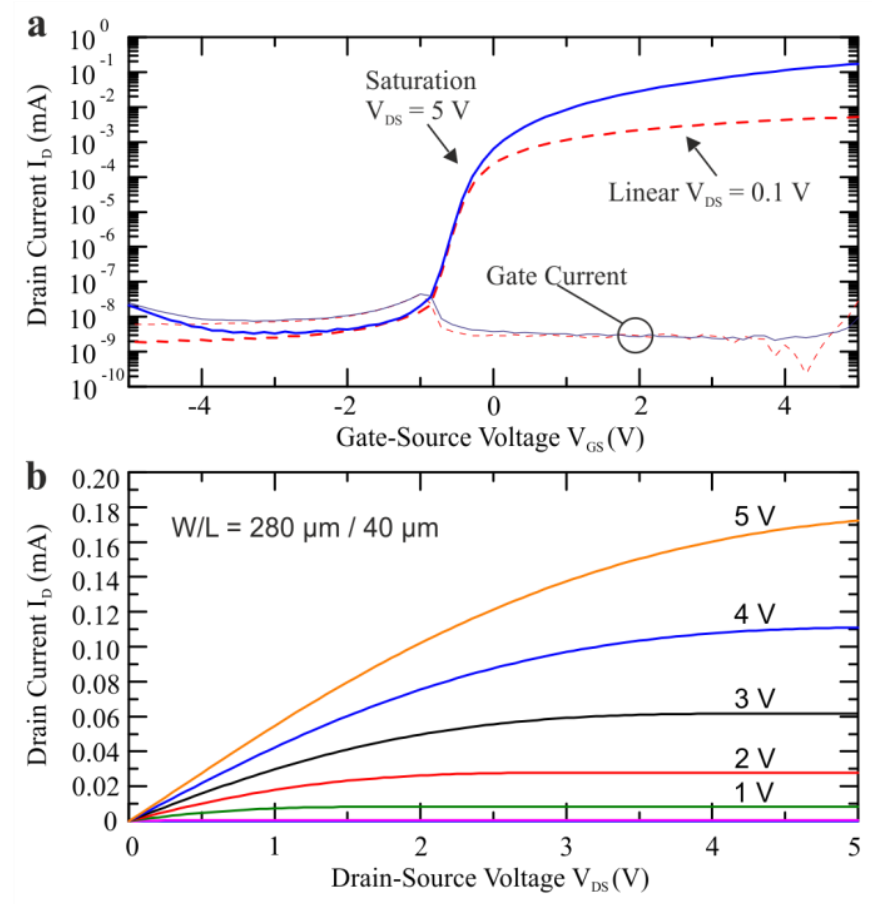

Fig. 3. TFT characteristics before irradiation and low temperature treatment. (a) Saturation $\left(\mathrm{V}_{\mathrm{DS}}=5 \mathrm{~V}\right)$ and Linear transfer $\left(\mathrm{V}_{\mathrm{DS}}=0.1 \mathrm{~V}\right)$ curves. (b) Output curves for Gate-Source voltages $\left(\mathrm{V}_{\mathrm{GS}}\right)$ ranging from $0 \mathrm{~V}$ to $5 \mathrm{~V}$.

\section{RESULTS}

\section{A. Transistor Performance}

Fig. 3 shows measured representative transfer and output curves for a virgin $280 \mu \mathrm{m}$ wide and $40 \mu \mathrm{m}$ long flexile TFT. From the saturation transfer curve (Fig. 3a), a threshold voltage $\left(V_{t h}\right)$ of $-0.16 \mathrm{~V}$, a field effect mobility $\left(\mu_{F E}\right)$ of $13.4 \mathrm{~cm}^{2} \mathrm{~V}^{-1} \mathrm{~s}^{-1}$, a subthreshold swing $(S)$ of $146 \mathrm{mV} / \mathrm{dec}$, a $I_{O N} / I_{O F F}$ ratio of $10^{7}$, and a maximum specific transconductance $g_{m} / \mathrm{W}\left(V_{G S}=5 \mathrm{~V}\right)$ of $0.23 \mathrm{~S} \mathrm{~m}^{-1}$, were extracted. The gate current of this device is $<10 \mathrm{pA}$. These values are in agreement with other high quality state-of-the-art a-IGZO TFTs [5].

\section{B. Electron Irradiation Effects}

The TFTs were exposed to electron irradiation with fluence ranging from $10^{6} \mathrm{e}^{-} / \mathrm{cm}^{2}$ to $10^{12} \mathrm{e}^{-} / \mathrm{cm}^{2}$. Fig. 4 a presents the averaged transfer curves from the same 25 transistors measured before and after exposure to electron irradiation at $10^{12} \mathrm{e} / \mathrm{cm}^{2}$. These curves are distinct from Fig. 3 due to the averaging process. From these measurements, a $V_{\text {th }}$ shift of $+0.09 \mathrm{~V} \pm 0.05 \mathrm{~V}$ was observed, whereas the $\mu_{F E}$ and $S$ decreased by $-0.6 \pm 0.5 \mathrm{~cm}^{2} \mathrm{~V}^{-1} \mathrm{~s}^{-1}$ and $-0.6 \mathrm{mV} /$ dec. The $I_{O N} / I_{O F F}$ ratio and the gate current were virtually unaffected by the electron irradiation, remaining at $10^{7}$ and $<10 \mathrm{pA}$, indicating that the $\mathrm{Al}_{2} \mathrm{O}_{3}$ gate insulator was not damaged. Fig. $4 \mathrm{~b}$ shows the averaged output curves for the same transistors, reflecting the decrease in the maximum $I_{D}$ due to the increase of $V_{t h}$ and the decrease of $\mu_{F E}$. Electron irradiation has no impact on the quality of the drain and source contacts, given than no current crowding effects are observed for low $V_{D S}$ [16]. The evolutions of both the $V_{t h}$ and $\mu_{F E}$ are shown in Fig. $4 \mathrm{c}$ and d, respectively, 
a

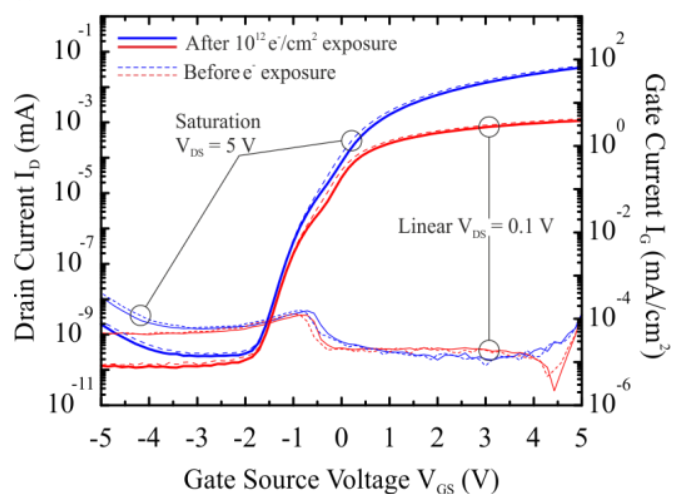

b

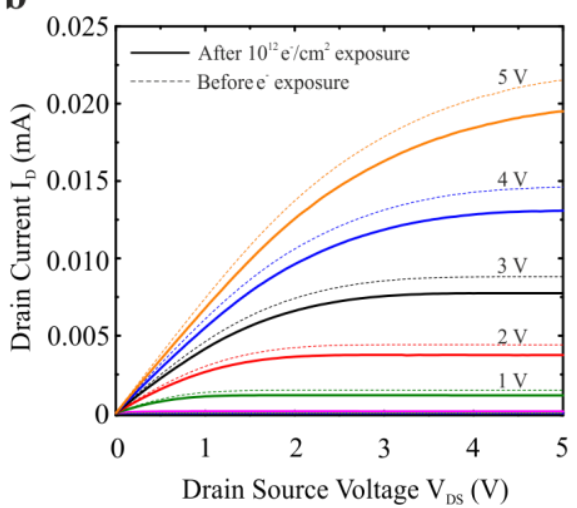

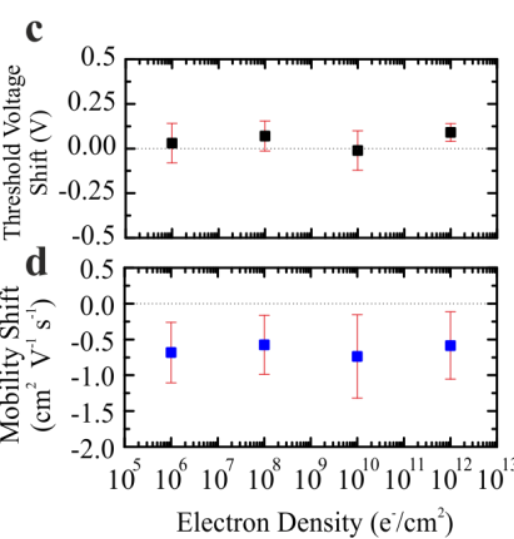

Fig. 4. Averaged transfer (a) and output (b) curves from 25 a-IGZO TFTs exposed to electron irradiation with a density of $10^{12} \mathrm{e}^{-} / \mathrm{cm}^{2}$. Evolution of the $V_{t h}(\mathrm{c})$ and the $\mu_{\mathrm{FE}}(\mathrm{d})$ for all electron irradiation densities.

with increasing electron irradiation. Parameter extraction was performed before and after irradiation on the same set of transistors (25 TFTs for each electron irradiation density). The threshold voltage is demonstrated to be virtually independent of the electron irradiation, whereas the mobility decreases after irradiation. This shift is significantly smaller than the $1 \mathrm{~V} V_{\text {th }}$ shift observed for rigid a-IGZO TFTs exposed to $0.8 \mathrm{MeV}$ electrons with a fluence of $10^{14} \mathrm{e}^{-} \mathrm{cm}^{-2}$ [10] and can be explained by the reduced interaction of the electrons with the low-density polymer substrate. Additionally, it is observed that the shift's magnitude of the mobility is independent of the irradiation fluence. The observed decrease of both $I_{D}$ and $\mu_{F E}$, as well as the increase of $V_{t h}$ can be explained by the formation of both oxygen interstitial and zinc vacancy acceptor defects, caused by the exposition to high energy electrons, as well as by charge trapping at the gate/dielectric. [17], [18].

\section{Low Temperature Measurements}

Next, the impact of low temperature on flexible a-IGZO transistors was investigated. Fig. 5 shows the averaged performance variation of the characterized transistors. Characteristic curves were extracted from 16 transistors. The reduced number of measurements is because the temperatureinduced expansion of the polyimide substrate, complicated reliable contacting of the devices, and different transistors had to be measured for each temperature. Fig. 5a shows the averaged output curves for the measured transistors, where a decrease of the drain current is observed for $78 \mathrm{~K}$ in comparison to the values observed at $310 \mathrm{~K}$. This is explained by the decrease of the thermal energy available for the thermal activation of electron trapped in defect sites. As it was observed for the irradiated transistors, no current crowding effect is observed for low $\mathrm{V}_{\mathrm{DS}}$ values. An average $V_{t h}$ of $0.607 \pm 0.002 \mathrm{~V}$ was extracted from the measured TFTs at $78 \mathrm{~K}$ (Fig. 5b), corresponding to a positive shift of $0.511 \mathrm{~V}$ when compared to the $0.097 \pm 0.06 \mathrm{~V}$ extracted from the same devices at room temperature. The evolution of $V_{t h}, S$ and $\mu_{F E}$ are presented in Fig. 5c, d and e, respectively. $V_{t h}$ increases for lower temperatures, whereas the subthreshold swing and $\mu_{F E}$ decrease for the same interval. Previous studies on rigid TFTs have presented similar trends for the $V_{t h}$, and $\mu_{F E}$. In addition, measurements down to $10 \mathrm{~K}$ on rigid a-IGZO TFTs demonstrated that the subthreshold swing increased for temperatures below $80 \mathrm{~K}$ [19], which was attributed to a change from band conduction to variable range hopping [20].

\section{Combined irradiation and temperature}

Finally, the combined influence of radiation and low temperatures was investigated, Fig. 6 presents the transfer curves of two transistors at $310 \mathrm{~K}$ and $78 \mathrm{~K}$. One device was irradiated with $10^{12} \mathrm{e}^{-} / \mathrm{cm}^{2}$, the reference TFT (inset) was not irradiated. As can be seen, the parameter shifts are only slightly affected by the applied electron irradiation. The irradiated sample exhibited $V_{t h}, \mu_{F E}$ and $S$ shifts of $+0.38 \mathrm{~V},-5.95 \mathrm{~cm}^{2} \mathrm{~V}^{-1} \mathrm{~s}^{-1}$ and $+30 \mathrm{mV} / \mathrm{dec}$, after being cooled down to $78 \mathrm{~K}$. The same parameters shifted by $+0.13 \mathrm{~V},-5 \mathrm{~cm}^{2} \mathrm{~V}^{-1} \mathrm{~s}^{-1}$ and $-21 \mathrm{mV} / \mathrm{dec}$ for the non-irradiated sample. The irradiated sample presents larger parameter shifts at low temperatures. Although these differences are small and could be related to intrinsic performance variations of the samples, the measurement indicates that a combined radiation-temperature effect in flexible a-IGZO TFTs has to be considered for space applications. This combined effect can be caused by an increased number of defects and traps in the irradiated transistors. The thermally activated occupation and de-occupation of these traps then changes the low temperature behavior of the TFTs.

\section{CONCLUSION}

The suitability of flexible a-IGZO TFTs was assessed for the development of electronic devices for conformable and lightweight space applications. Electron irradiation followed by low temperature treatment down to $78 \mathrm{~K}$ were conducted to simulate the harsh environment found in Low Earth Orbit. Trap creation caused by electron irradiation induces a positive $V_{t h}$ shift and a decrease of the $\mu_{F E}$ for electron irradiation densities up to $10^{12} \mathrm{e}^{-} / \mathrm{cm}^{2}$ and electron energy of $34.1 \mathrm{MeV}$. Nonetheless, the variation of the electron irradiation fluence did not influence the magnitude of these shifts. Subsequent low temperature measurements down to $78 \mathrm{~K}$ resulted in an average decrease of $\mu_{F E}$ of $20 \%$, accompanied by a positive $V_{t h}$ shift and a decrease of the subthreshold swing that reached a minimum 

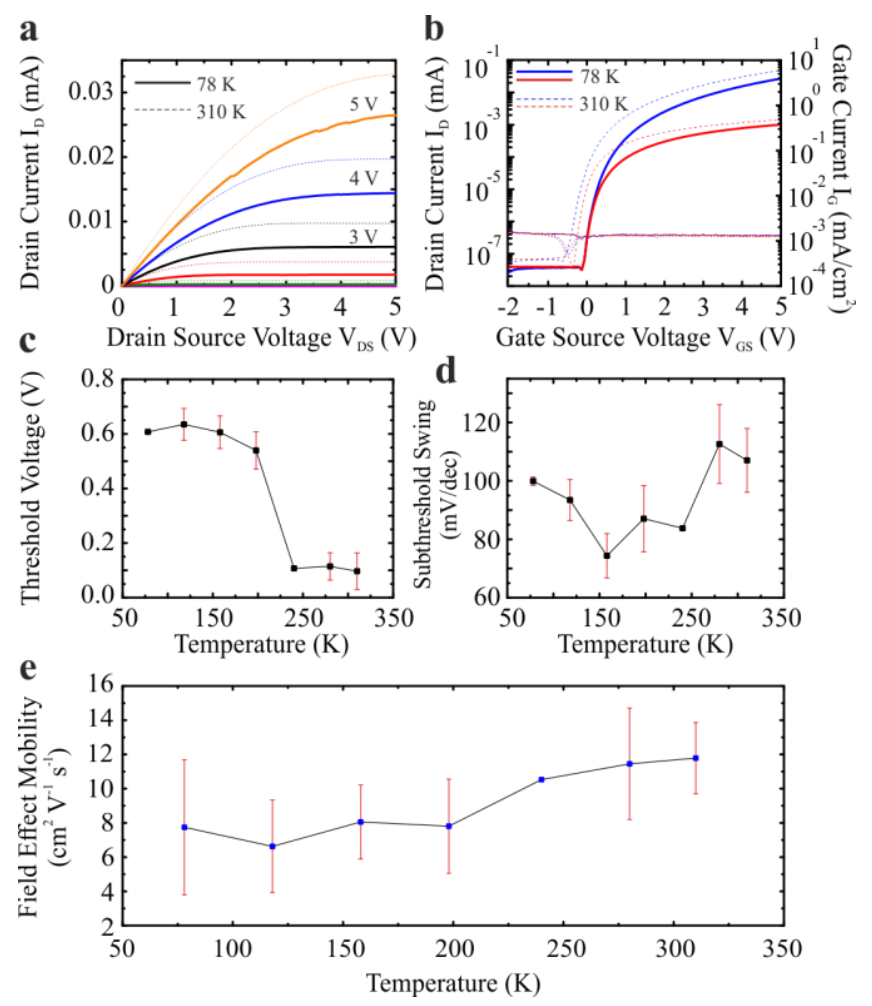

Fig. 5. Low temperature measurements from a total of 16 transistors. (a) Output curves at $78 \mathrm{~K}$ and $310 \mathrm{~K}$. (b) Transfer curves. (c)-(e) $V_{t h}, S$ and $\mu_{F E}$ variation for temperatures from $78 \mathrm{~K}$ to $310 \mathrm{~K}$.

of $66.5 \mathrm{mV} / \mathrm{dec}$. Furthermore, it was demonstrated that the temperature induced parameter shifts are slightly influenced by previous electron irradiation. This showed that a-IGZO TFTs fabricated on flexible substrates are a viable option for the development of lightweight and unobtrusive devices for space applications, and future smart textiles for space suits.

\section{REFERENCES}

[1] T. Dachev, W. Atwell, E. Semones, B. Tomov, and B. Reddell, "Observations of the SAA radiation distribution by Liulin-E094 instrument on ISS," Adv. Sp. Res., vol. 37, no. 9, pp. 1672-1677, 2006.

[2] F. J. Pavón-Carrasco and A. De Santis, "The South Atlantic Anomaly: The Key for a Possible Geomagnetic Reversal," Front. Earth Sci., vol. 4, no. April, pp. 1-9, 2016.

[3] E. G. Stassinopoulos and J. P. Raymond, "The space radiation environment for electronics," Proc. IEEE, vol. 76, no. 11, pp. 1423-1442, 1988.

[4] K. Nomura, H. Ohta, A. Takagi, T. Kamiya, M. Hirano, and H. Hosono, "Room-temperature fabrication of transparent flexible thin-film transistors using amorphous oxide semiconductors," Nature, vol. 432, no. 7016, pp. 488-492, 2004.

[5] L. Petti et al., "Metal oxide semiconductor thin-film transistors for flexible electronics," Appl. Phys. Rev., vol. 3, no. 2, p. 21303, 2016.

[6] J. C. Costa, A. Wishahi, A. Pouryazdan, M. Nock, and N. Münzenrieder, "Hand-Drawn Resistors, Capacitors, Diodes, and Circuits for a Pressure Sensor System on Paper," Adv. Electron. Mater., vol. 1700600, pp. 1-8, 2018.

[7] M. Nag et al., "Flexible AMOLED display and gate-driver with selfaligned IGZO TFT on plastic foil," Dig. Tech. Pap. - SID Int. Symp., vol. 45 , no. 1, pp. 248-251, 2014.

[8] D. Karnaushenko et al., "Biomimetic Microelectronics for Regenerative Neuronal Cuff Implants," Adv. Mater., vol. 27, no. 43, pp. 6797-6805, 2015.

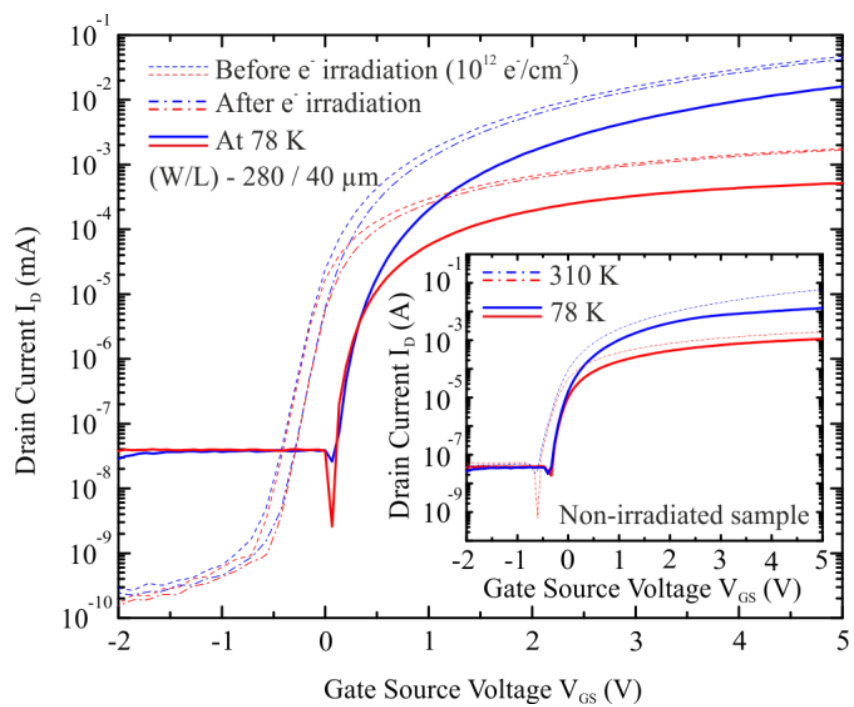

Fig. 6. Evolution of the saturation and linear transfer curves for a transistor irradiated with the highest electron density, followed by exposure to $78 \mathrm{~K}$.

[9] P. G. Bahubalindruni et al., "Analog circuits with high-gain topologies using a-GIZO TFTs on glass," IEEE/OSA J. Disp. Technol., vol. 11, no. 6, pp. 547-553, 2015.

[10] H. J. Moon, S. H. Jung, M. K. Ryu, K. I. Cho, E.-J. Yun, and B. S. Bae, "Effect of high-energy electron beam irradiation on the gate-bias stability of IGZO TFTs," J. Korean Phys. Soc., vol. 60, no. 2, pp. 254-260, 2012.

[11] N. Münzenrieder, L. Petti, C. Zysset, T. Kinkeldei, G. A. Salvatore, and G. Tröster, "Flexible self-aligned amorphous InGaZnO thin-film transistors with submicrometer channel length and a transit frequency of 135 MHz," IEEE Trans. Electron Devices, vol. 60, no. 9, pp. 1-6, 2013.

[12] J. G. Funk and G. F. Sykes, Jr., "The Effects of Simulated Space Environmental Parameters on Six Commercially Available Composite Materials," NASA Tech. Pap. 2906, 1989.

[13] F. Gabriel et al., "The Rossendorf radiation source ELBE and its FEL projects," Nucl. Instruments Methods Phys. Res. Sect. B Beam Interact. with Mater. Atoms, vol. 161-163, pp. 1143-1147, 2000.

[14] Technical Report No. TRS-381, "The Use of Parallel Ionization Chambers in High Energy Electron and Photon Beams," IAEA (International Atomic Energy Agency), Vienna 1997.

[15] H. Shichman and D. Hodges, "Modeling and simulation of insulated fieldeffect transistor switching circuits," Solid-State Circuits, vol. 3, no. 3, pp. 285-289, 1968.

[16] K. Takechi, M. Nakata, T. Eguchi, H. Yamaguchi, and S. Kaneko, "Study on current crowding in the output characteristics of amorphous InGaZnO4 thin-film transistors using dual-gate structures with various active-layer thicknesses," Jpn. J. Appl. Phys., vol. 48, no. 8 Part 1, pp. 08160610816066, 2009.

[17] S. H. Jeong, B. S. Bae, K. M. Yu, M. K. Ryu, K. I. Cho, and E.-J. Yun, "Properties of IGZO thin films irradiated by electron beams with various energies," J. Korean Phys. Soc., vol. 61, no. 6, pp. 867-872, 2012.

[18] G. K. Dayananda, R. C. Shantharama, A. Jayarama, and H. J. Kim, "Study of radiation resistance property of a- IGZO thin film transistors," 2016 IEEE Int. Conf. Recent Trends Electron. Inf. Commun. Technol. RTEICT 2016 - Proc., vol. 2, no. 4, pp. 1816-1819, 2017.

[19] M. D. Hossain Chowdhury, P. Migliorato, and J. Jang, "Low temperature characteristics in amorphous indium-gallium-zinc-oxide thin-film transistors down to $10 \mathrm{~K}$," Appl. Phys. Lett., vol. 103, no. 15, pp. 1-5, 2013.

[20] J. Jeong, G. Jun Lee, J. Kim, S. Moon Jeong, and J. H. Kim, “Analysis of temperature-dependent electrical characteristics in amorphous In-Ga-Zn$\mathrm{O}$ thin-film transistors using gated-four-probe measurements," J. Appl. Phys., vol. 114, no. 9, 2013. 